\title{
Significados de conflicto cotidiano y la construcción de paz en jóvenes y adultos mayores de Bucaramanga' ${ }^{12}$
}

\author{
Ara Mercedes Cerquera Cordoba \\ Doctora en Ciencias Psicológicas \\ Universidad Pontificia Bolivariana, Colombia \\ Correo electrónico: ara.cerquera@upb.edu.co

\section{Yeferson Jahir Matajira Camacho \\ Especialista en Psicología Clínica Universidad Pontificia Bolivariana, Colombia Correo electrónico: yeferson.matajira@upb.edu.co}

Arbey Jair Peña Peña

Psicólogo

Universidad Pontificia Bolivariana, Colombia Correo electrónico: arbey.pena94@gmail.com

\section{Margie Stefania Quintero Mantilla \\ Especialista en Psicología Clínica Universidad Pontificia Bolivariana, Colombia Correo electrónico: margie.quintero@upb.edu.co \\ Carol Juliana García Vargas \\ Psicóloga \\ Universidad Pontificia Bolivariana, Colombia

Recibido: 27/03/2019

Evaluado: 24/09/2019

\section{Resumen}

El objetivo del presente estudio es explorar los significados acerca del conflicto y la construcción de paz presentes en un grupo de jóvenes y adultos mayores de la ciudad de Bucaramanga, Santander, Colombia. Con un enfoque cualitativo, diseño fenomenológico y muestra no probabilística constituida por 22 jóvenes y 20 adultos mayores, se utilizó una entrevista a profundidad diseñada y validada por el semillero de investigación por medio de la cual se abordaron cuestiones relacionadas al conflicto cotidiano y la construcción de paz. Como resultados, se encontró que tanto los jóvenes como los adultos mayores hicieron énfasis en definir el conflicto como situación "problema" que trae desacuerdos entre las partes involucradas. Respecto a los mecanismos de resolución, prevaleció el diálogo. Por último, se logra identificar que los adultos mayores guardan mayor esperanza frente a la posibilidad de alcanzar la paz en comparación con los jóvenes.

Palabras clave

Significados, Adulto mayor; Joven; Conflicto; Construcción de paz.

12 Para citar este artículo: Cerquera, A., Matajira, Y., Peña, A., Quintero, M. y García, C. (2021). Significados del conflicto y la construcción de paz en jóvenes y adultos mayores de Bucaramanga. Informes Psicológicos, 21(1), 183-200 http://dx.doi.org/10.18566/infpsic.v21n1a12 


\title{
Meanings of the conflict and peacebuilding in youth and older adults of Bucaramanga
}

\begin{abstract}
The objective of this study is to explore the meanings about conflict and peacebuilding present in a group of young people and older adults from the city of Bucaramanga, Santander, Colombia. With a qualitative approach, a phenomenological design and a non-probabilistic sample made up of 22 young people and 20 older adults, an in-depth interview designed and validated by the research hotbed was used, through which issues related to daily conflict and peace building were addressed. As a result, it was found that both young people and older adults defined conflict as a "problem" situation, which brings disagreements between the parties involved. Regarding resolution mechanisms, dialogue prevailed. Finally, it is possible to identify that older adults have greater hope towards the possibility of achieving peace compared to young people.
\end{abstract}

Keywords

Meanings, older adult, youth, conflict, peace building.

\section{Significados do conflito e da construção da paz em jovens e idosos de Bucaramanga}

\section{Resumo}

0 objetivo deste estudo é explorar os significados sobre conflito e construção da paz presentes em um grupo de jovens e idosos da cidade de Bucaramanga, Santander, Colômbia. Com abordagem qualitativa, desenho fenomenológico e amostra não probabilística composta por 22 jovens e 20 idosos, utilizou-se entrevista em profundidade desenhada e validada pelo grupo de pesquisa, por meio da qual abordaram-se questões relacionadas ao conflito cotidiano e à construção de Paz. Como resultado, constatou-se que tanto os jovens quanto os idosos enfatizam a definição do conflito como uma situação "problema" que traz divergências entre as partes envolvidas. Em relação aos mecanismos de resolução, o diálogo prevaleceu. Por fim, é possivel identificar que os idosos têm maior esperança quanto à possibilidade de alcançar a paz em relação aos jovens.

Palavras chave Significados, idoso, jovem, conflito, construção da paz. 


\section{ntroducción}

Las sociedades a lo largo de su evolución se han visto permeadas por situaciones de conflicto que en su mayoría ha desembocado en manifestaciones de violencia. Hemos pasado por un sinnúmero de guerras civiles e intervenciones militares, originadas en su mayoría por desacuerdos entre las partes protagonistas o luchas de poder. Pero el conflicto se encuentra inmerso también en la sociedad a partir de manifestaciones dentro de diferentes contextos: la familia, la escuela, el trabajo, el barrio o comunidad, entre otros.

En este sentido, Colombia no solo ha pasado por casi 60 años de violencia armada y conflictos, influidos por aspectos políticos, económicos y sociales, sino también, año a año, se aumentan las cifras de violencia intrafamiliar, homicidios, acoso escolar o laboral, entre otras. Para el caso de Santander, se registraron a 2018 un saldo de 894 muertes violentas, $y$, para el caso de Bucaramanga, se señalaron 228 de muertes violentas y 2.459 casos de violencia interpersonal, 443 de violencia intrafamiliar, 668 casos de violencia de pareja y 335 exámenes médico legales por presunto delito sexual, según el informe Forensis 2018 (Medicina Legal y Ciencias Forenses, 2018), siendo el municipio de Santander con más casos relacionados a estas categorías.

Estas cifras tan sólo dan cuenta de una parte de las recurrentes manifestaciones de violencia en el departamento. Es claro que muchos casos permanecen en el silencio, ya sea por miedo o por naturalización de las distintas manifestaciones de violencia en la sociedad. A partir de esto, se expone la necesidad de analizar la realidad de las manifestaciones violentas y la noción acerca de los conflictos y sus representaciones e imaginarios en la sociedad Santandereana, de manera que, a partir de ello, se aporte a la construcción de estrategias de intervención eficaces.

Como es sabido, a lo largo de la historia, el conflicto se ha entendido como una situación de confrontación entre dos partes opuestas o en contradicción, asociada a conductas, emociones, pensamientos y sentimientos generalmente incompatibles que imposibilitan un cambio positivo entre los individuos en desacuerdo (Calderón, 2009; Fuquen, 2003; Romero, 2003), en ocasiones lleva a agresiones mutuas para anular al contrario, trayendo consigo sentimientos de frustración o irritación entre los involucrados (Ortega, López, \& Álvarez, 2007; Simmel, 2010; Womack, 1988). En este sentido, París (2005) lo describe como expresión de incompatibilidad entre individuos o grupo de personas que realizan acciones "que implican el ejercicio del poder de uno sobre otro en un ambiente de ausencia, libre interpretación o trasgresión de normas" (p. 32).

Ahora, en relación a la forma como se interpreta el conflicto, Romero (2003) describe los conflictos como funcionales o disfuncionales. Los primeros son los que promueven la creatividad, la resolución de problemas, adaptación a nuevas condiciones y el trabajo en equipo; mientras que los disfuncionales son aquellos en donde las partes se ven afectadas severamente, trayendo consigo reacciones violentas y destructivas. 
Por tanto, el conflicto es un proceso dinámico, una construcción social que puede ser experimentada de forma positiva o negativa según cómo se aborde y se le dé fin, con posibilidades de ser transformado y superado con o sin ayuda de terceros (Fuquen, 2003).

Además, es importante resaltar que generalmente los conflictos gestionados inadecuadamente desembocan en violencia directa, estructural o cultural, las cuales tienden a permear la cultura, la religión, las ideologías, el lenguaje y el arte (Calderón, 2009; Galtung, 1969).

Ahora, teniendo en cuenta que la violencia y el conflicto hacen parte inevitable de la vida (Palacios \& Safford, 2002; Pécault, 2001), y, a pesar de existir múltiples mecanismos de gestión positiva de los conflictos, muchas sociedades han elegido la agresión como mecanismo de resolución (Muñoz, Herrera, Molina, \& Sánchez, 2005), siendo este el caso de Colombia, pues los conflictos socio-políticos han generado cambios al entramado social. Estas manifestaciones, al ser normalizadas, legitiman la violencia, de alli la necesidad de entender los comportamientos conflictivos, gestionarlos y transformarlos en una perspectiva positiva que promuevan el ejercicio de la paz como construcción cultural.

Sin embargo, la presencia de conflictos cotidianos que afectan las relaciones intrapersonales, interpersonales y sociales (generadores de malestar), implica, a la par, el anhelo o búsqueda de bienestar, el cual desde la perspectiva del sentido común se entiende como paz; pero resulta muy importante comprender que la paz no necesariamente se constituye por la ausencia de conflictos, sino también con el acceso a la educación para la paz (Paz sin fronteras, 2011); de manera que la construcción de paz se relaciona con las acciones que se orientan a identificar y apoyar estructuras que promueven el fortalecimiento de la paz para evitar una recaída a las manifestaciones de conflicto (Organización de las Naciones Unidas, 1995). Además, esta construcción de paz se logra trabajando por un objetivo en común encaminado a terminar con las violencias e impactar en la cultura, para transformarla hacia comportamientos de paz (Hernández, 2008; Rettberg, 2012).

Así pues, al hablar de cultura de paz, se debe entender que la cultura "encierra el conocimiento adquirido que las personas utilizan para interpretar su experiencia y generar comportamientos" (Zaragoza, 2010, p. 155); y la paz se entiende como algo mucho más profundo que la simple ausencia de guerra (Fisas, 2011), frente a la cual se necesita del "ejercicio diario, [...] en el que asumimos la igualdad de todos los seres en dignidad, el reconocimiento mutuo de los derechos, y la voluntad de construir juntos una ciudadanía común, encarando los conflictos de manera dialógica" (De Oña Cots \& García, 2016, p. 116).

De acuerdo con Fisas (1998), la cultura de paz es entendida como un proceso relacionado con la superación, reducción o evitación de todo tipo de violencias, donde las situaciones de conflicto pueden ser oportunidades creativas, de encuentro, comunicación y cambio; entonces, esto implica crear una nueva cultura, que integre discursos, valores, representaciones y actitudes al servicio de unas relaciones intergrupales más justas y solidarias (Barreto, Borja, Serrano, \& López, 2009; Fernández, 1999) que permitan plantear 
mecanismos de acción ante los nuevos retos que presenta la naturalidad del proceso de transformación del conflicto-paz (Rettberg, 2003; Schultze-Kraft, 2012).

El presente estudio se llevó a cabo con el objetivo de explorar los significados acerca del conflicto y la construcción de paz presentes en un grupo de jóvenes y adultos mayores de la ciudad de Bucaramanga, Santander, Colombia. Lo anterior, partiendo de que para lograr un acercamiento a los escenarios de realidad social que el sujeto habita, es necesario comprender los significados que los seres humanos otorgan a sus experiencias, prácticas y acciones cotidianas (Hernández, 2003; Lacasa, Vélez \& Sánchez, 2005) pues estos dan la posibilidad al ser humano para que influya y sea influido a través de la subjetividad implícita en la relación con los otros, es decir, la construcción social deviene de la praxis de los significados como reflejo de la vida social (Arcila, Mendoza, Jaramillo \& Cañon, 2010; Salas-Menotti, 2008). Es importante aclarar que para esta investigación las narrativas constituyeron el medio central para plasmar los significados en torno al conflicto cotidiano y la construcción de paz que ambas puntas generacionales poseen.

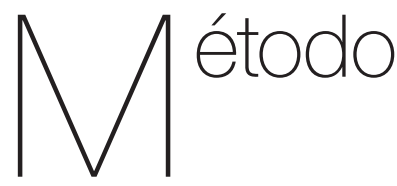

\section{Diseño}

Se empleó un enfoque cualitativo a través de un diseño de tipo fenomenológico para indagar sobre los diferentes significados en torno a las temáticas de conflicto cotidiano y construcción de paz.

\section{Participantes}

La población estuvo constituida por jóvenes y adultos mayores de Bucaramanga y su área metropolitana. Se empleó un muestreo no probabilístico, a conveniencia e intencional, seleccionando un total de 42 participantes, subdivididos en 20 adultos mayores y 22 jóvenes. Los criterios de inclusión fueron: Sub-muestra vejez: Ser mayor de 60 años de edad y residir en Bucaramanga o su área metropolitana, ausencia de limitaciones determinantes en habla y/o audición y brindar el consentimiento de participación y firma de autorización para la realización de la entrevista. Sub-muestra Juventud: Estar en el rango de los 11 y 18 años de edad, ser estudiante de bachillerato de las instituciones de Bucaramanga y su área metropolitana, brindar Consentimiento de participación y firma de autorización (director de las instituciones educativas en caso de ser menor de edad).

\section{Instrumentos}

Entrevista a profundidad construida por el Semillero Calidad de Vida en la Tercera Edad de la Universidad Pontificia Bolivariana, seccional Bucaramanga. Dicho instrumento abordó cuestiones relacionadas con las ideas, percepciones, expectativas y alternativas frente al conflicto cotidiano y la construcción de paz. La entrevista a profundidad posibilita un acercamiento directo a la vida del otro, precisa su subjetividad y otros aspectos fundamentales de sus perspectivas frente al mundo y a sí mismo (Ballesteros, 2005). Dicho instrumento fue evaluado por jueces expertos de las áreas de psicología social, psicología educativa y psicopedagogía, dando una calificación cualitativa acerca del mismo, el cual, 
posterior a las correcciones pertinentes, se considera un instrumento válido para evaluar las categorías presentadas.

\section{Consideraciones éticas}

Para la presente investigación se guardó la confidencialidad de los participantes mediante la firma del documento del consentimiento informado en el cual también se daba claridad sobre los objetivos y características de la investigación como lo determina la Ley 1090 de 2006, numeral 9 del artículo 2.

\section{Procedimiento}

La investigación se dividió en cuatro fases: (1) Contacto con instituciones educativas, centros geriátricos y selección de la muestra; (2) Aspectos éticos y autorización de las entidades e instituciones; (3) Aplicación y exploración de significados; y (4) Categorización de la información y análisis de resultados, donde se realizó el proceso de categorización de las narrativas mediante el software de análisis cualitativo Atlas ti. (v.7.5). Los temas se categorizaron por medio de códigos y las categorías organizadas de forma deductiva. Las categorías de análisis fueron: percepción y conocimiento del conflicto, consecuencias del conflicto, alternativas de solución del conflicto, percepción sobre la paz, alternativas y expectativas para la construcción de la paz

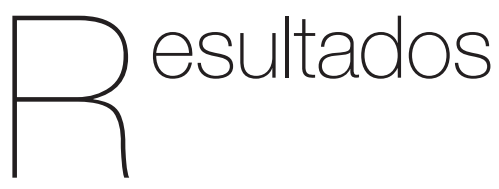

Para el análisis del discurso de los participantes se establecieron a partir de un proceso de codificación abierta, dos categorías genéricas denominadas "Conflicto cotidiano" y "Paz y construcción de paz", con las subcategorías descritas en la Figura 1.

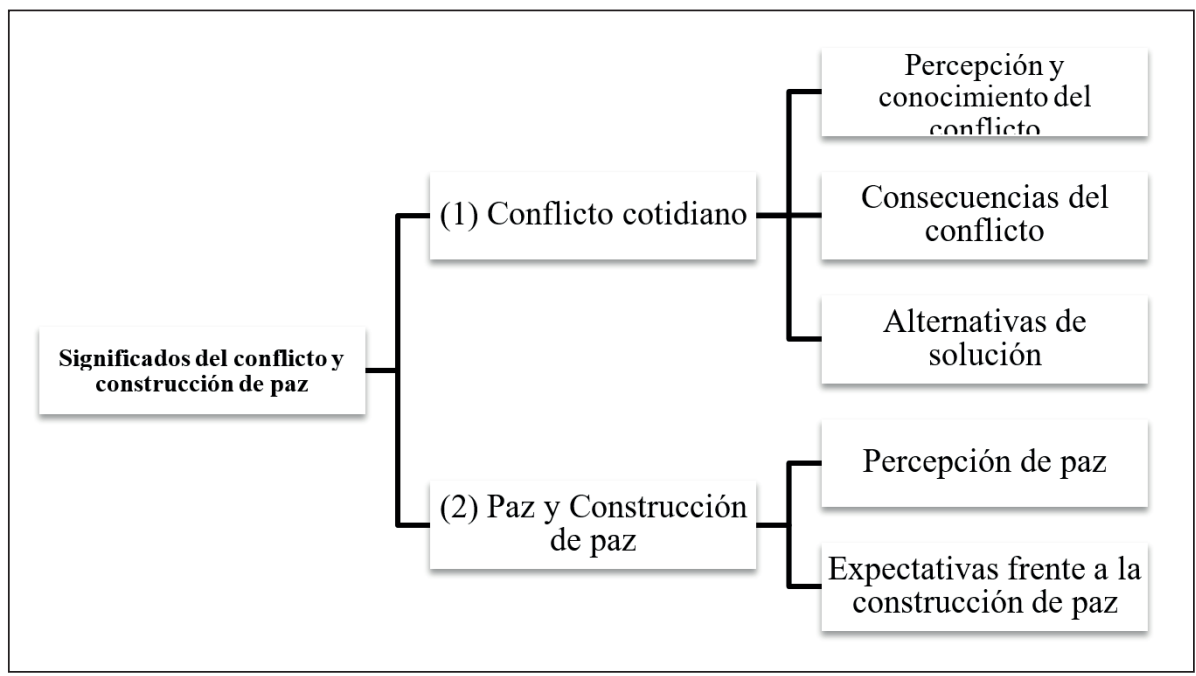

Figura 1. Esquema categorial de Significados del conflicto cotidiano y la construcción de paz en una muestra de jóvenes y adultos mayores de Bucaramanga, Santander. 


\section{Conflicto cotidiano}

Tabla 1.

Sub-categoría Percepción y conocimiento del conflicto cotidiano en jóvenes y adultos mayores de Bucaramanga

Percepción y conocimiento del conflicto cotidiano

\begin{tabular}{|c|c|}
\hline Jóvenes & Adultos Mayores \\
\hline $\begin{array}{l}\text { - Situación problema generada por el desacuerdo } \\
\text { o discusión entre dos o más personas }\end{array}$ & $\begin{array}{l}\text { - Desacuerdo o discusión entre dos o más } \\
\text { personas: ámbito conyugal, familiar y/o laboral }\end{array}$ \\
\hline - Asociado a la pelea, agresión y calumnia & - Asociación al conflicto armado \\
\hline $\begin{array}{l}\text { - Experiencia personal asociada a la violencia directa } \\
\text { en contexto escolar, comunitario y/o familiar }\end{array}$ & $\begin{array}{l}\text { - Asociación a dificultades económicas y/0 } \\
\text { falta de oportunidades laborales }\end{array}$ \\
\hline $\begin{array}{l}\text { - Identificación de formadores y contextos generadores de } \\
\text { herramientas y alternativas frente a los conflictos cotidianos }\end{array}$ & - Asociación a falta de educación \\
\hline
\end{tabular}

Fuente: Autoría propia

Inicialmente, los jóvenes participantes hicieron énfasis en definir el conflicto cotidiano como situación "problema", que genera dificultades en la consecución de metas o satisfacción de necesidades.

Por su parte, la población mayor refirió que un conflicto es una discusión o desacuerdo entre dos o más personas, sea en el matrimonio o relación de pareja, con los hijos o demás miembros de la familia o en el trabajo, atribuido por la mayoría a falta de comunicación, desinformación o intolerancia; se evidencia en afirmaciones como:

"...es cuando nadie se pone de acuerdo o pelean por cualquier cosa", "son los problemas que se nos presentan día a día".

Así mismo, el término "conflicto" se asocia con el conflicto armado colombiano en expresiones como: "el conflicto de la guerra, de los guerrilleros y del gobierno", "pues, el conflicto es, supuestamente, como una secuencia de una guerra civil".

También a dificultades económicas y/o falta de oportunidades laborales, y falta de educación, tal y como se refiere en las siguientes verbalizaciones:

"porque si el esposo no tiene trabajo y la señora tampoco, ahí es donde hay el mayor conflicto, cómo hacen para darle la educación a un muchacho, si no hay ninguna solución", "Conflicto entre situación económica también...porque nos ha tocado luchar, viajar de una parte a otra, y siempre se han presentado conflictos que generan mucha violencia y eso afecta la situación económica". 
Tabla 2.

Sub-categoría percepción de consecuencias del conflicto cotidiano en jóvenes y adultos mayores de Bucaramanga

\begin{tabular}{|c|c|}
\hline \multicolumn{2}{|c|}{ Consecuencias del conflicto cotidiano } \\
\hline Jóvenes & Adultos Mayores \\
\hline - Generador de afectividad negativa & - Vínculos distantes entre las personas \\
\hline - Daño a relaciones y vínculos interpersonales & - Daño físico o psicológico a otros \\
\hline - Promotor de unidad y liderazgo & - Actos ilegales \\
\hline
\end{tabular}

Fuente: Autoría propia

Por otra parte, como "consecuencias" adscritas al desarrollo de este tipo de conflictos, los jóvenes exponen principalmente el "daño a las relaciones interpersonales", el "rencor" y sensación de "desconfianza" como efectos negativos; sin embargo, como efectos positivos rescatan que en medio de los conflictos suele generarse y promoverse la "unidad" a la par que se gestionan o se da el "surgimiento de liderazgos", lo cual permite el fortalecimiento de relaciones interpersonales y el empoderamiento.

Por su parte, los adultos mayores afirman que lo percibido como conflicto genera consecuencias en las relaciones interpersonales, hacer daño a las personas involucradas en la disputa o que surjan conductas violentas, situaciones de caos y/o actos ilegales, tal como se afirma en las siguientes verbalizaciones:

"Ios conflictos pueden llevar a la perdición a la gente, porque no tiene control con nadie, entonces hacen lo que se la dan la gana", "entonces la falta de diálogo pues, se presta para que hayan malos entendidos o la situación no haya solución a los problemas sino que día a día se empeoren".

Tabla 3.

Sub-categoría Alternativas de solución al conflicto cotidiano en jóvenes y adultos mayores de Bucaramanga

\begin{tabular}{ll}
\hline \multicolumn{2}{c}{ Alternativas de solución al conflicto cotidiano } \\
\hline \multicolumn{1}{c}{ Jóvenes } & \multicolumn{1}{c}{ Adultos Mayores } \\
\hline & $\bullet$ Garantía de derechos \\
- Herramientas para la resolución de conflictos: & - Justicia Social \\
Arbitraje, Consejería y Negociación en la política \\
& - Espiritualidad: Oración. \\
\hline
\end{tabular}

Fuente: Autoría propia 
En cuanto a ideas de solución a los conflictos, para los jóvenes son el arbitraje, la consejería y la negociación, lo cual representa la pretensión de resolver la situación de la manera más justa posible. Esta solución hace parte de la idealización de los jóvenes en la forma en que se pueden tomar decisiones para la resolución de diferentes conflictos.

Seguido a esto, frente a la subcategoría de alternativas de solución a los conflictos, los adultos mayores hicieron énfasis en la garantía de los derechos de acceso a la educación y al trabajo digno, la inversión en el sistema de salud, en narrativas como

"...que haya educación y oportunidades para todos. Es muy difícil ir a decir que va a haber educación, que se va a acabar la pobreza; la pobreza genera violencia".

Así mismo, los adultos mayores referían la justicia social como una posibilidad de solución, denotándose en afirmaciones como:
"Necesitamos un gobierno, no como piensan muchos que es comunismo, pero si, que seamos una sociedad donde se pueda compartir, y si yo no tengo y tú tienes, nos ayudemos".

Agregando a lo anterior, para los gerontes toma importancia significativa el diálogo, pues favorece el acuerdo entre las partes implicadas en el conflicto. Así mismo, el afrontamiento religioso a través de la oración, que se constituyen como posibles soluciones ante los conflictos.

"Eso síya es cuestión del gobierno... lo único que puede hacer uno como ciudadano es orar mucho por la paz, pedirle a Dios que les dé mucha sabiduría, para que ellos puedan realmente, esa gente de buen corazón, que verdaderamente quiera la paz ...".

\section{Paz y construcción de paz}

Tabla 4.

Sub-categoría significados acerca de la paz en jóvenes y adultos mayores de Bucaramanga

\begin{tabular}{|c|c|c|}
\hline \multicolumn{3}{|c|}{ Percepción de paz } \\
\hline & Jóvenes & Adultos Mayores \\
\hline & $\begin{array}{l}\text { Asociación con emociones y conductas como amor, alegría, } \\
\text { respeto y No violencia }\end{array}$ & $\begin{array}{l}\text { - Asociación a estados de tranquilidad, armonía, sosiego y } \\
\text { espiritualidad. }\end{array}$ \\
\hline & Cualidades de las relaciones que representan paz & - Asociación al sentimiento de amor \\
\hline & Estados físicos y/o mentales asociados a la paz & - Garantía de derechos fundamentales \\
\hline & Consecución de la paz & \\
\hline
\end{tabular}

Fuente: Autoría propia 
Frente a los significados de paz, los jóvenes la asocian a la tranquilidad y serenidad, sentimientos de amor y felicidad, y valores como la tolerancia y buena convivencia, tal como los expresan en las siguientes afirmaciones:

"Con estar un poco más tranquilo, estar en un ambiente donde la convivencia sea bonita", "tranquilidad", "la paz es serenidad, amor, principalmente eso, amor".

Del mismo modo, lo relacionaban con la ausencia de guerra o de hechos delictivos, asociado a la desaparición de los grupos armados:
"La Paz es, no sé, es viajar a cualquier parte del país sin tener temor de que lo secuestre o nos asesinen, estar tranquilos de que los campesinos no se van a ver afectados y que desaparezcan esos grupos", "la paz es cuando no roban, no matan, es muy distinta la paz que uno conoce como la verdadera paz".

Se puede evidenciar en los discursos de los sujetos la tranquilidad y la serenidad que deja el pasar de los años, al asociar la paz con el amor y sentimientos espirituales que se reflejan en una experiencia y sabiduría de los mayores.

Tabla 5.

Sub-categoría Expectativas frente a la construcción de paz en jóvenes y adultos mayores de Bucaramanga

\begin{tabular}{lll}
\hline \multicolumn{2}{c}{ Expectativas frente a la construcción de paz } & \multicolumn{1}{c}{ Adultos Mayores } \\
\hline \multicolumn{1}{c}{ Jóvenes } & Positivismo frente a la idea de paz \\
\hline $\begin{array}{l}\text { Contradicción entre perspectiva respecto a la construcción } \\
\text { de paz y expectativas frente a la consolidación } \\
\text { del mismo como proceso social progresivo }\end{array}$ & $\begin{array}{l}\text { Desesperanza respecto al concebir o materializar } \\
\text { acciones para la construcción de paz }\end{array}$ \\
& $\begin{array}{l}\text { Posibilidad de paz: Compromiso del } \\
\text { estado y ética en la política. }\end{array}$ \\
\hline
\end{tabular}

Fuente: Autoría propia

En la subcategoría de expectativas para la construcción de paz surgió una contradicción, ya que la mayoría de los jóvenes manifestaban poseer una visión positiva frente a la posibilidad de que existan acciones para construir paz, sin embargo, se evidenció una desesperanza marcada en la creencia de que esas acciones se puedan llevar a cabo en Colombia; así se expresó en las siguientes afirmaciones:

“...Puesyo, laverdad, personalmente, no creo en el proceso de paz ni en las soluciones, porque siempre va a haber algo o alguien que quiere hacer daño por interés o por querer dañar a las comunidades", "que cada vez en el país todo está peor".

Lo anterior también se evidenció en las narrativas de los adultos mayores, donde la mayoría refería poseer una visión positiva para la construcción de paz, pero una marcada dificultad para concebir o materializar dichas acciones, evidenciándose en verbalizaciones como: 
"si, pero, entonces yo creo que nunca va a haber paz, yo no creo que nunca va a haber paz en este mundo".

Además, se encuentra en el discurso de los adultos la posibilidad de paz ligada a una política con mayor criterio moral y ético; esto se denota en las siguientes afirmaciones:

"...sería poner gente en el gobierno, de verdad, que sea honrada, que sea de buena fe, que no vea la política como un negocio".

Tabla 6.

Sub-categoría Alternativas para la construcción de paz en jóvenes y adultos mayores de Bucaramanga

\begin{tabular}{lll}
\hline & \multicolumn{1}{c}{ Alternativas para la construcción de paz } \\
\hline Jóvenes & & \multicolumn{1}{c}{ Adultos Mayores } \\
\hline Vinculación de valores éticos & - & Fomento de valores: solidaridad y respeto \\
$\begin{array}{l}\text { Generación de vínculos caracterizados } \\
\text { por la disponibilidad de ayuda, } \\
\text { pretensión de unidad y no violencia }\end{array}$ & - & Promover la paz desde el hogar \\
& - & Cambios individuales: conciencia ciudadana \\
& - Participación en acciones colectivas: huelgas y manifestaciones. \\
& - & Promover la paz a través de la espiritualidad \\
\hline
\end{tabular}

Fuente: Autoría propia

Finalmente, en esta subcategoría, los jóvenes afirmaron que los valores éticos inherentes al ser humano como la tolerancia, el respeto a la diferencia, la honestidad, compasión y la aceptación de la pluralidad son alternativas para la construcción de paz, y los expresaron de esta manera:

\section{"escuchar, brindar una ayuda cuando seanecesario", "quelagente sea respetuosa, sea tolerante..." "siendo honesto y ayudando a las demás personas que no tengan los mismos recursos que yo".}

Así mismo refirieron que se puede aportar a la construcción de paz desde cambios individuales: "pues yo como estudiante pues estudiar y ser buena persona para poder...mmm estudiar algo para poder aportar", "pues en este momento sería estudiar para que más adelante pudiese investigar de cómo hacer la paz".

Por otra parte, los adultos mayores proponen el uso de valores como la solidaridad y el respeto:

"...que seamos una sociedad en la que se pueda compartir, donde, si yo no tengo y tú tienes, nos ayudemos...", sin pensar en ofender a nadie, ni tratar mal a otro".

De igual forma, mencionan que otra de las alternativas se da propiamente generando paz en el núcleo familiar: 
"la paz empieza por la casa, sin peleas", "Primero que todo ponerme en paz con la familia, eh... no se presenten roces con ninguno, con toda la familia esté uno en paz".

Coincidiendo con que los cambios personales son alternativas para la construcción de paz, desde los cambios individuales y la generación de nuevas ideas; esto se pudo evidenciar en afirmaciones como:

"La paz la hace uno mismo, la paz la hace uno", "la paz debe ser algo muy interior para uno, estar en paz con uno mismo, con la familia, vecinos, amigos".

Así mismo, los adultos afirmaron que la paz se puede promover a través de la oración y la transmisión de conocimiento y esperanza a los jóvenes, lo cual expresaron de esta manera:

"Pues si en la familia los hijos son seres de bien que se han criado con valores que nunca tienen problema con nadie, son unas personas que también pueden aportar a la paz ..." "el aporte es con la familia, con los hijos, que los hijos sean realmente buenos ciudadanos, que lo que hagan lo hagan legalmente, que sean legales en todo, tener buenas costumbres", "... importante hacer mucha oración, y orar muchísimo por el país que haya la paz porque Dios es el único realmente... si todo el mundo orara yo creo que sí se lograría la paz, pidiéndole a Dios eso"

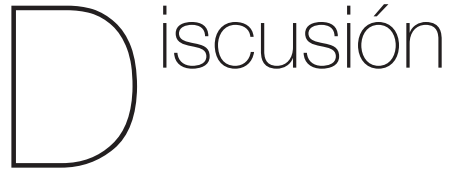

De cara a la subcategoría denominada "percepción de consecuencias del conflicto cotidiano en jóvenes y adultos mayores de Bucaramanga", desde la perspectiva de los jóvenes, el conflicto se concibe básicamente como la consecuencia generada por un enfrentamiento entre dos partes que se perciben como obstáculos para la consecución de metas, intereses u objetivos; siendo una percepción negativa del conflicto, donde las agresiones resultan, al parecer, frecuentes en los contextos escolares, comunitarios y familiares; sin embargo, para el caso de los adultos mayores, el conflicto se asocia con la presencia de desacuerdos y discusiones en el ámbito familiar y laboral. Lo anterior permite evidenciar que existe una prevalente percepción del ser humano como agresivo por naturaleza, generando limitaciones en diversos contextos frente a la posibilidad de trascender éste como "impasse" (Jares, 1977; Martín \& Calvo, 2013; Paris, 2005).

En este mismo sentido, los jóvenes reconocen que los contextos escolares, comunitarios y familiares son, a su vez, espacios promotores o reforzadores de herramientas y alternativas que permiten a través del reconocimiento del otro, del aprendizaje y la comprensión de la diversidad de pensamientos, gestionar o solucionar los conflictos para así disminuir el sufrimiento, el malestar o la injusticia (Jares, 1977; Martín \& Calvo, 2013; Paris, 2005). Lo descrito permite corroborar, tal como lo menciona Galtung (1998), que, en la medida que se cuentan con 
competencias y habilidades para gestionar los conflictos de forma creativa y no violenta, se construyen relaciones y estructuras más inclusivas y sostenibles (Barbero, 2006).

Vale destacar que los adultos mayores entrevistados relacionan en reiteradas oportunidades el concepto de conflicto con el fenómeno de violencia armada que ha vivido el país y, desde allí, desarrollan sus valoraciones e ideas. Esto puede deberse a que de forma directa o indirecta la población colombiana se ha visto inmersa en este fenómeno que data con más de 50 años (Centro Nacional de Memoria Histórica, 2013). Además, desde su independencia, Colombia ha soportado manifestaciones violentas que han dejado en el país cifras gigantescas de muertes y un sentimiento generalizado de desconfianza, desesperanza, dolor y desequilibrio (Jaime, 2011); por ende, las representaciones sociales y significados asociados al conflicto han de verse permeados por la realidad a la cual los colombianos han estado adscritos.

Por otra parte, entre las "consecuencias de los conflictos" (subcategoría) señaladas por los jóvenes, se resalta el daño a las relaciones interpersonales, desembocando en sentimientos de rencor y desconfianza; de similar manera, los adultos mayores describen afectación a los vínculos interpersonales, con presencia de daños físicos o psicológicos y acontecimiento de actos ilegales; lo anterior da cuenta de la prevalente visión negativa del conflicto, puesto que tanto adultos mayores como jóvenes hacen énfasis en las tensiones y emociones negativas provocadas por el conflicto (Jares, 1977; Martín \& Calvo, 2013; Paris, 2005). Así que, estas percepciones son propias de la etapa denominada "escalada" de los conflictos, esperándose que posteriormente suceda el estancamiento o la desescalada (Ruiz, 2006).

Ahora, en relación a la subcategoría "percepción acerca de las alternativas de solución a los conflictos cotidianos", el hecho de que los jóvenes señalan el arbitraje, la consejería y negociación como herramientas para hacer frente a los conflictos cotidianos y la reconciliación, da cuenta de lo que París (2005) resalta como herramientas necesarias de cara a la transformación de los conflictos, a saber: el reconocimiento, el empoderamiento, el diálogo y el afecto. Por su parte, los adultos mayores han mencionado que las alternativas para solucionar los conflictos cotidianos son de orden más pragmático y estructural, haciéndose especial énfasis en el papel del Estado, la dimensión espiritual y la capacidad de diálogo entre los individuos. Corroborando entonces que, desde la perspectiva de los adultos mayores, la percepción o disposición frente a los conflictos de orden intrapsíquico, interpersonal y entre personas e instituciones, se puede desembocar como un proceso de transformación (Lederach, 1996, citado por Paris, 2005; Fisas, 1998; 2011; Paris, 2005).

En referencia a los significados acerca de la paz, los jóvenes ligan la paz a cierto estado de satisfacción y/o bienestar afectivo, el cual se encuentra exento de la relación con otras personas, es decir, solo ciertas verbalizaciones se orientan a comprender la paz como una construcción interpersonal o con lo que, desde la concepción positiva del conflicto, se define como la diversidad de pensamientos, la empatía y el empoderamiento (Jares, 1977; Martín, \& Calvo, 2013; Paris, 2005). En ese orden 
de ideas, Fisas (1987) considera que la paz, en sentido amplio, supone la ausencia de violencia directa y estructural; ello implica generar bienestar, satisfacción de las necesidades humanas básicas, identidad y libertad para todos.

Para los adultos mayores, el significado de la paz se relaciona con la satisfacción de necesidades básicas, el equilibrio socioeconómico, el acceso a la salud, a la educación, a la vivienda y al empleo para todos los integrantes de la sociedad. Lo anterior, lo afirman en sintonía con lo referenciado por Galtung (1998) y Fisas (1998), citados en Tovar y Sacipa (2010), quienes conciben la paz estructural como condiciones de la estructura social que permiten el libre desarrollo de los miembros de la comunidad y, por tanto, propenden por la dignidad humana.

Respecto a las expectativas frente a la construcción de paz, se evidencia una marcada contradicción, pues jóvenes y adultos mayores coinciden al mostrar una visión positiva frente a acciones para construir paz, sin embargo, manifiestan incredulidad frente a que éstas se puedan llevar a cabo en el país, relacionando esta posibilidad de paz a una política con mayor criterio moral y ético. Lo anterior guarda relación con lo expuesto por Ramos-Vidal, Holgado, y Maya-Jariego (2014), quienes hallaron que la población colombiana percibe inoperancia, confabulación, o, en muchos casos, corrupción política por parte de los representantes del gobierno.

De cara a las alternativas para la construcción de paz, el hecho de que algunos jóvenes y adultos mayores afirman no poseer un conocimiento de alternativas para la construcción de paz, deja en evidencia que la lectura que hacen de ellos mismos los relega como sujetos al margen del proceso social que enfrenta su país. Sin embargo, también se logra identificar otra tendencia señalada que se anida en la lectura de la construcción de paz relacionada con el conflicto armado colombiano, resultando dos alternativas contradictorias entre sí, a saber: una perspectiva guerrerista desde la cual el objetivo es "eliminar" a ciertos actores del conflicto a través del ejercicio de la fuerza armada, y un enfoque conciliador basado en delimitar o evitar que el estado ejerza violencia.

Por tanto, como lo destaca Andrade (2012), resulta fundamental que se vincule como herramienta para la construcción de paz a la memoria, ya que ésta constituye el fundamento del perdón y la reconciliación, siendo además un factor de concienciación, transformación, organización y fortalecimiento social, sea cual sea el origen del conflicto y las características del mismo. Así, la educación para la paz, ha de ser un esfuerzo capaz de consolidar una nueva manera de ver, entender y vivir el mundo, desde el propio ser y continuando con los demás. De manera que propicie un clima de confianza y seguridad a las personas y a las sociedades, superando desconfianzas, ayudando a movilizarlas y a sobrellevar sus diferencias (Fisas, 2011; Garaigordobil, \& Maganto, 2011; Hernández, 2010).

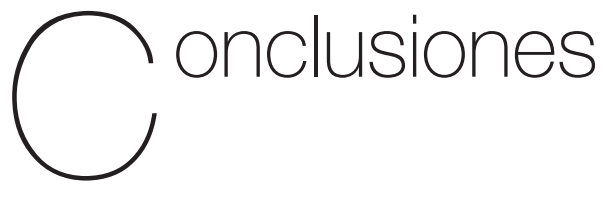

A modo de cierre, respecto a la categoría "conflicto cotidiano" se puede concluir que la muestra de jóvenes y adultos 
mayores entrevistados consideran que el conflicto es una situación negativa que desemboca en violencia y acontece en los diversos contextos, siendo evidente la necesidad de fomentar la adquisición de competencias y habilidades para que los ciudadanos logren empoderarse de la gestión adecuada de los conflictos, de manera creativa y bajo el principio de no violencia, pero esto deberá estar basado en una nueva interpretación que permita a los jóvenes y adultos concebir el conflicto como una oportunidad o espacio de transformación positiva.

En relación a la categoría Paz y construcción de paz, se concluye que, en primer lugar, respecto al significado en torno a la paz, los jóvenes la relacionan con satisfacción y/o bienestar afectivo desde un punto de vista individual y los adultos mayores con la satisfacción de necesidades básicas (económicas, de salud, educación, vivienda y al empleo); ambos grupos generacionales conciben la paz alejada de una construcción grupal o en sociedad.

También, tanto jóvenes como adultos mayores, coinciden en la necesidad de construir espacios de paz, sin embargo, muestran descontento o incredulidad con que estos espacios se logren dar en el país, puesto que, a su parecer, es necesaria política con mayor criterio moral y ético. Finalmente, algunos jóvenes y adultos mayores afirman no poseer un conocimiento de alternativas para la construcción de paz, dando cuenta de una posición al margen del proceso social de construcción del país, transitando entre una perspectiva guerrerista desde la cual el objetivo es "eliminar" a ciertos actores del conflicto y una conciliadora basada en delimitar o se ejerza violencia.
Lo visto hasta aquí permite dar cuenta de la necesidad de poner en marcha acciones sociales que propendan por la educación y acompañamiento en torno a gestión positiva de conflictos, reconcillación y cultura para la paz, desde un punto de vista intergeneracional. Puesto que, si bien el tamaño muestral de la presente investigación es reducido, da luces acerca de que pensamos como sociedad en torno a los conflictos y las limitantes que tenemos a la hora de gestionarlos de forma positiva; sabiendo que el aprendizaje frente a conflictos pasados y el entrenamiento en la transformación de los mismos, servirá como herramienta preventiva de conflictos futuros que desemboquen en todo tipo de violencias.

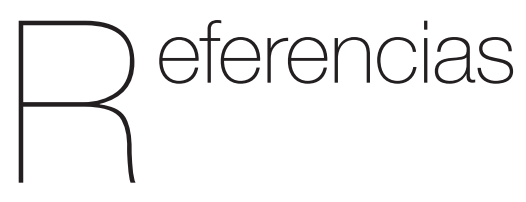

Andrade, O. (2012). Memoria y construcción de paz. En Víctimas: miradas para la construcción de paz. Serie documentos para la paz, 2, 15-43. Bogotá: Universidad Jorge Tadeo Lozano, Observatorio de construcción de paz y fondo para la sociedad Civil Colombianos por la Paz, FOS.

Arcila M. P. A., Mendoza R. Y. L., Jaramillo, J. M., \& Cañón Ortiz, Ó. E. (2010). Comprehension of the meaning from Vygotsky, Bruner and Gergen. Diversitas: Perspectivas en psicología, 6(1), 37-49.

Ballesteros, B. (2005). El concepto de significado y el análisis del comportamiento 
y otras perspectivas. Universitas Psychologica, 4(2), 231-244.

Barbero, A. (2006). Construyendo la paz en medio de la guerra: Colombia. Escola de Cultura de Pau. Recuperado de http:// escolapau.uab.cat/img/programas/ colombia/colombia020e.pdf

Barreto, I., Borja, H., Serrano, Y., \& López, W. (2009). La legitimación como proceso en la violencia política, medios de comunicación y construcción de culturas de paz. Universitas Psychologica, 8(3), 737-748.

Calderón, P. (2009). Teoría de conflictos de Johan Galtung. Revista de paz y conflictos, 2, 60-81.

Centro Nacional de Memoria Histórica (2013). ¡Basta ya! Colombia: memorias de guerra y dignidad, informe general. Recuperado de http://www.centrodememoriahistorica. gov.co/descargas/informes2013/bastaYa/ basta-ya-colombia-memorias-de-guerray-dignidad-2016.pdf

De Oña Cots, J. \& García, A. (2016). Proyecto Escuela: Espacio de Paz. Reflexiones sobre una Experiencia en un Centro Educativo. Revista Electrónica Iberoamericana Sobre Calidad, Eficacia Y Cambio En Educación, 14(2), 115-131.

Fernández, I. (1999). Prevención de la violencia y resolución de conflictos. Madrid, España: Nancea S.A. de Ediciones.

Fisas, V. (1987). Introducción al estudio de la paz y de los conflictos. Barcelona: Lerna.

Fisas, V. (1998). El sexo de la violencia: género y cultura de la violencia. Barcelona: Icaria.
Fisas, V. (2011). Educar para una cultura de paz. Quaderns de Construcció de Pau, 20, 7-24.

Fuquen, M. (2003). Los conflictos y las formas alternativas de solución. Tabula Rasa, 1, 265-278.

Galtung, J. (1969). Violence, Peace, and Peace Research. Journal of Peace Research, 6(3), 167-191.

Galtung, J. (1998) Tras la violencia, 3R: reconstrucción, reconciliación, resolución. Afrontando los efectos visibles e invisibles de la guerra y la violencia. Colección. País Vasco, España: Red Gernika, BakeazGernika.

Garaigordobil, M., \& Maganto, C. (2011). Empatía y resolución de conflictos durante la infancia y la adolescencia. Revista Latinoamericana de Psicología, 43(2), 255-266.

Hernández, E. (2008). La Paz imaginada por quienes la construyen: Iniciativas Civiles de Paz de Base Social identifican sus sueños de Paz. Reflexión Política, 10(9), 134-147.

Hernández, E. (2010). Aproximación Teórica a los Significados de la Mediación en Conflictos Armados. Reflexión Política, 12(24), 28-40.

Hernández, F. (2003). El constructivismo como referente de las reformas educativas neoliberales. Educere, 7(23), 433-440. Recuperado de https://www.redalyc.org/ pdf/356/35602317.pdf

Jaime, M. (2011). El conflicto armado en Colombia. Revista de Derecho, (19), 119-125. 
Jares, X. (1977). El lugar del conflicto en la organización escolar. Revista Iberoamericana de Educación, (15), 53-73.

Lacasa, P., Vélez, R., \& Sánchez, S. (2005). Objetos de aprendizaje y significado. Revista de educación a Distancia, 4(5). Recuperado de: https://revistas.um.es/red/ article/view/24421

Martín, J., \& Calvo, G. (2013). Perseguir el sueño o caer en lo que existe: la socialización del maestro novel entre el" habitus" y la investigación-acción. Revista Interuniversitaria de Formación del Profesorado, (78), 137-144.

Medicina Legal y Ciencias Forenses (2018). Forensis, datos para la vida: Herramienta para la interpretación, intervención y prevención de lesiones de causa externa en Colombia. Recuperado de http://www.medicinalegal. gov.co/documents/20143/386932/ F o rens i s +2018 . p d f / be4816a4-3da3-1ff0-2779-e7b5e3962d60

Muñoz F., Herrera J., Molina B., \& Sánchez S. (2005). Investigación de la paz y los Derechos Humanos desde Andalucía. Granada, España: Imprenta Comercial Motril

Organización de las Naciones Unidas. (1995). Supplement to an Agenda for Peace: Position Paper of the Secretary-General on the Occasion of the Fiftieth Anniversary of the United Nations. UN

Ortega, M., López, S., \& Álvarez, M. (2007). Los conflictos. En "autores", Ciencias Psicosociales. España: Universidad de Cantabria. Recuperado de https://ocw. unican.es/pluginfile.php/1420/course/ section/1836/tema_09.pdf
Palacios, M., \& Safford, F. (2002). Colombia: país fragmentado, sociedad dividida. Bogotá, Colombia: Grupo Editorial Norma.

París A., S. (2005). La transformación de los conflictos desde la filosofía para la paz. Tesis doctoral. Universidad Jaume I de Castellón de la Plana, España.

Paz sin fronteras. (2011). La paz es un derecho humano. Recuperado de http:// pazsinfronteras.org/

Pécault, D. (2001). Guerra contra la sociedad. Bogotá, Colombia: Planeta.

Ramos-Vidal, I., Holgado, D., \& Maya-Jariego, I. (2014). Las redes personales de los desplazados internos por la violencia política en Colombia: una aproximación al caso del departamento del Atlántico. Trace, (65), 51-68.

Rettberg, B. (2003). Diseñar el futuro: una revisión de los dilemas de la construcción de paz para el postconflicto. Revista de Estudios Sociales, (15), 15-28.

Rettberg, B. (2012). Construcción de paz en Colombia. Bogotá, Colombia: Ediciones Uniandes.

Romero, S. (2003). Teoría del conflicto social. En "Autor" Negociación directa y asistida. Lima, Perú: ASOPDES. Recuperado de http://abacoenred.com/wp-content/ uploads/2015/10/Teor\%C3\%ADa-delconflicto-social-ASOPDES-2003.pdf

Ruiz, Y. (2006). Análisis y resolución de conflictos desde una dimensión psicosocial. Recuperado de http://repositori.uji.es/ xmlui/bitstream/handle/10234/78608/ forum_2006_14.pdf 
Salas-Menotti, I. (2008). Significado psicológico de la violencia y la agresión en una muestra urbana colombiana. Diversitas: Perspectivas en Psicología, 4(2), 331-343.

Schultze-Kraft, M. (2012). La cuestión militar en Colombia: la fuerza pública y los retos de la construcción de la paz. Construcción de Paz en Colombia, 405-433.

Simmel, G. (2010). El conflicto sociología del antagonismo. Madrid, España: Ediciones Sequitur.
Tovar, C., \& Sacipa, S. (2010). Significados e interacciones de paz de jóvenes integrantes del grupo "Juventud Activa" de Soacha, Colombia. Universitas Psichologia, 10(1), $35-46$

Womack, D. (1988). Assessingthe ThomasManagement Communication Quartely, 1(3), 321-349.

Zaragoza, L. (2010). Cultura, identidad y etnicidad, aproximaciones al entorno multicultural: rompiendo costumbres y paradigmas cotidianos. Cuicuilco, 17(48), 149-164. 